\title{
Germline selection for deleterious mutation in males
}

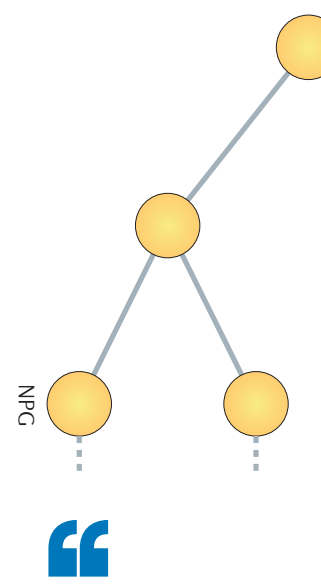

how do we explain why the mutation rate of the MEN2B substitution is severalhundred-fold higher than for the rest of the genome?

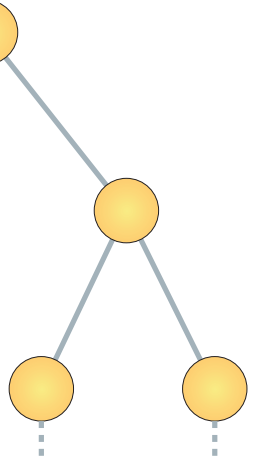

For a number of intriguing monogenic conditions, the causative mutation occurs predominantly de novo in the male germline and is transmitted at a markedly increased rate with advancing paternal age. An investigation of one such human condition - namely, multiple endocrine neoplasia type $2 \mathrm{~B}$ (MEN2B) - offers an explanation for this atypical inheritance pattern: spermatogonial stem cells with the mutation gain a proliferative advantage in the testes and thus have a selective advantage over normal cells.

MEN2B is an aggressive thyroid cancer syndrome that, in virtually all new sporadic cases, is caused by the same substitution mutation $(2,943 \mathrm{~T} \rightarrow \mathrm{C})$ in the $R E T$ protooncogene and is transmitted by fathers whose average age is greater than the average paternal age. How do we account for this inheritance pattern, and how do we explain why the mutation rate of the MEN2B substitution is several-hundredfold higher than for the rest of the genome? Two explanations can be advanced: either that the causative nucleotide is a mutation hotspot or that the mutation is under positive selection in the testes.

To distinguish between these models, the authors studied the spatial distribution of the $2,943 \mathrm{~T} \rightarrow \mathrm{C}$ RET mutation in the testes of 14 normal men across three age groups (namely, 19-23 years, 36-68 years and 75-80 years). Each testis was divided into 192 segments and assessed by a PCR-based protocol for the frequency of the MEN2B-causative RET mutation. In the middle-aged group, the RET mutation frequency was higher than the genome-wide average; clusters of segments were visible in which mutations occurred at a much higher frequency than in others. By contrast, the youngest group of men had very few mutations. These results are consistent with a paternal age effect and also indicate that the mutations seen in the middle group were probably not occurring until after puberty. The mutation pattern seen in the oldest sample was heterogeneous, although this discrepancy was removed when the mutation frequency was corrected for age-related cell death.
Computational simulations were then used to identify a cellular mutation model that best captures all of the parameters observed in the data: the average mutation frequency seen across a testis; a measure of the spatial clustering of mutations in different segments across the testis; and the fraction of segments that falls under a threshold mutation frequency. The hotspot model was rejected, but the selection model was a good match to the data. In particular, the model was most consistent with a process in which the mutant spermatogonial cells occasionally divide symmetrically (shown in yellow in the figure) - rather than asymmetrically (shown in blue in the figure), as is normally the case. Although an asymmetrical division gives rise to another stem cell and a sperm progenitor, a symmetrical cell division would generate two stem cell daughters and therefore yield a much larger pool of genetically identical - in this case, mutated - cells.

Similar investigations could now be applied to study similarly inherited de novo disease mutations. Given what is known about the requirement for RET signalling in spermatogonial self-renewal in the mouse and the effect of the $2,943 \mathrm{~T} \rightarrow \mathrm{C}$ mutation in human tissue culture, the most likely explanation for the observed selective advantage seen in MEN2B is that the causal mutation weakens the strength of signalling of downstream pathways, shifting the balance between symmetric and asymmetric divisions.

Tanita Casci

ORIGINAL RESEARCH PAPER Choi, S.-K. et al. Positive selection for new disease mutations in the human germline: evidence from the heritable cancer syndrome multiple endocrine neoplasia type 2B. PLoS Genet. 8, e1002420 (2012). FURTHER READING Arnheim, N. \& Calabrese, P. Understanding what determines the frequency and pattern of human germline mutations. Nature Rev. Genet. 10, 478-488 (2009) 